\title{
05 | De las estructuras laminares a las estructuras metálicas en la arquitectura de Félix Candela. Análisis y reconstitución de la sala de exposiciones para el concurso del Palacio Olímpico de los Deportes, México 1968. From laminated structures to metal structures in the architecture of Félix Candela. Analysis and reconstitution of the exhibition hall for the competition for the Olympic Sports Centre, Mexico 1968 _Federico Luis del Blanco García, Ismael García Ríos
}

\section{Introducción}

En 1968 Félix Candela sorprendió al mundo con la construcción del Palacio de los Deportes para las olimpiadas de México. El proyecto original presentaba, junto a la cúpula construida, un edificio anexo que serviría como sala de ferias y exposiciones. Este se generaba a partir de la repetición de un módulo formado por una estructura arbórea que sostenía 144 fragmentos de paraboloides hiperbólicos. [1]

Lo realmente sorprendente es que ambos proyectos -la cúpula y su edificio anexo- distaban mucho de las estructuras laminares que otorgaron fama internacional a Félix Candela. [2] [3]

Durante dos décadas Candela se había especializado en la realización de estructuras laminares de hormigón con geometría parabólico-hiperbólica. En los años previos a la construcción del Palacio de los Deportes, Candela ya había ejecutado centenares de cubiertas de este tipo por todo México, destinándose la mayor parte de ellas a edificios industriales y siguiendo la geometría de paraguas invertido formado a partir de 4 paraboloides hiperbólicos.

El motivo principal que permitió a Candela llevar a cabo tal cantidad de proyectos fue el coste reducido que tenían los mencionados paraguas invertidos: la doble curvatura que aporta la geometría del paraboloide hiperbólico permitía reducir la cantidad de hormigón necesario para generar la cubierta. Además, como los paraboloides hiperbólicos son superficies regladas, los encofrados podían llevarse a cabo a partir de tablas de madera rectas. Sin embargo, la realización de estos encofrados requería de gran cantidad de mano de obra.

El panorama económico de México cambió cuando en 1964 Gustavo Díaz Ordaz llegó a la presidencia del país estableciendo unos salarios mínimos para los trabajadores. Como consecuencia de ello las estructuras laminares tuvieron un incremento de coste que forzó a Candela a buscar nuevas alternativas y dejar atrás sus famosas cubiertas.

El Palacio de los Deportes y su sala de exposiciones anexa son la materialización de los experimentos que Candela llevó a cabo durante los años previos y que tendrán una línea de continuación en proyectos posteriores.

[2] Alzado de la cúpula y la sala de exposiciones. SEGUi BUENAVENTURA, Miguel. Félix Candela arquitecto: exposición, depósito Elevado del Canal de Isabel II. MOPTMA, Instituto Juan de Herrera Madrid, 1994.

[3] Planta y alzado de la cúpula y la sala de exposiciones. SEGUI BUENAVENTURA, Miguel. Félix Candela arquitecto: exposición depósito Elevado del Canal de Isabel II. MOPTMA, Instituto Juan de Herrera. Madrid, 1994.

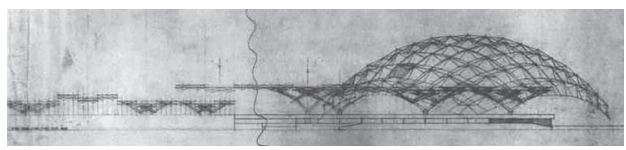

[2]

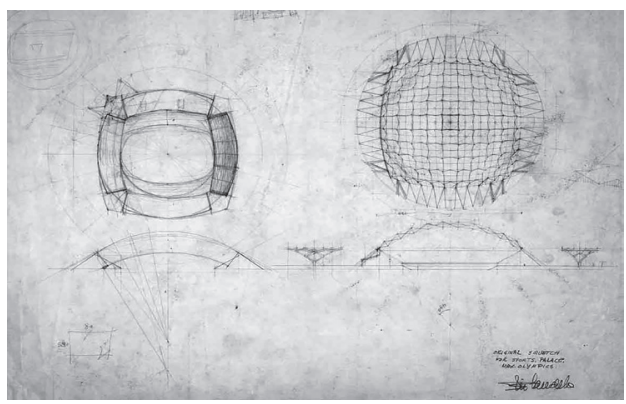

[3]

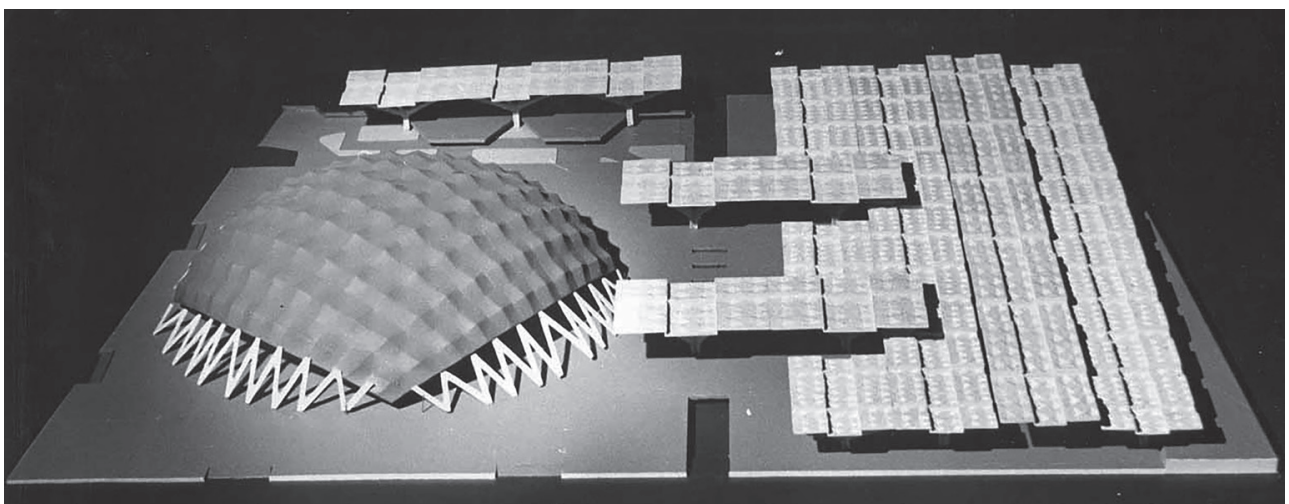

[1]
Resumen pág 63 | Bibliografía pág 68

Federico Luis del Blanco García. Arquitecto por la UPM (2009) y Profesor de postgrado en la misma Universidad. Profesor del Máster universitario oficial en Comunicación Arquitectónica, ETSAM, UPM. Miembro de la comisión académica del Curso de Especialización: Infografía. Diseño Multimedia para la Comunicación Arquitectónica. Máster en teoría, historia y análisis de arquitectura y especialista en diseño y animación 3D. federicodelblanco@ hotmail.com

Ismael García Ríos. Doctor Arquitecto por la UPM (1996) y Profesor Titular de la misma Universidad (2001). Docencia e investigación en Geometría y Dibujo de Arquitectura. Profesor responsable de la asignatura Taller Experimental de Infografía y del módulo instrumental del Máster universitario oficial en Comunicación Arquitectónica, ETSAM, UPM. Director del Curso de Especialización Infografía. Diseño Multimedia para la Comunicación Arquitectónica. ismael. garcia@upm.es

Palabras clave

Félix Candela, Frei Otto, Piñero, geometría, reconstitución 3D, estructura, hypar.

Keywords

Félix Candela, Frei Otto, Piñero, geometry, 3D reconstruction, structure, hypar 
La cúpula para el estadio Olímpico de México no fue la primera cúpula que diseñó Félix Candela ni sería la última, aunque sí fue la única que se llegó a construir.

Junto a ella la sala de exposiciones anexa estaba formada por los icónicos paraguas invertidos que Candela había desarrollado durante años. Sin embargo, estos se apoyan ahora sobre una compleja estructura arbórea metálica que permite sostener hasta 36 paraguas invertidos.

La cúpula y la sala de exposiciones estarán fuertemente influenciadas tanto por los arquitectos Frei Otto y Emilio Pérez Piñero como por sus asociados Praeger-Kavanagh-Waterbury.

\section{Tránsito de las estructuras laminares a las estructuras metálicas}

Tras el cambio realizado en los salarios mínimos, Félix Candela podía prever que la construcción de los 'cascarones' de hormigón en México tenía un fin próximo, al menos bajo las condiciones en las que se habían venido realizando.

Ese mismo año Félix Candela presenta una propuesta para el concurso del Crystal Palace en Londres con una estructura metálica que imita una forma arbórea y que repetirá para la zona de ferias y exposiciones del Palacio de los Deportes de México. Podemos apreciar el comienzo de un cambio no solo en el material empleado, sino también en la concepción arquitectónica. Las estructuras metálicas podrían ser la alternativa a los 'cascarones' de hormigón. [4] [5] [6] [7]

Tres años antes, en julio de 1961, Félix Candela acude a Londres con motivo del Congreso de la Unión Internacional de Arquitectos (UIA), donde le conceden el premio Auguste Perret y es nombrado miembro del jurado para un concurso de estudiantes bajo el tema de un teatro desmontable. Los ingenieros Ove Arup y Buckminster Fuller formarán junto con Candela el jurado del mismo ${ }^{1}$.

[4]

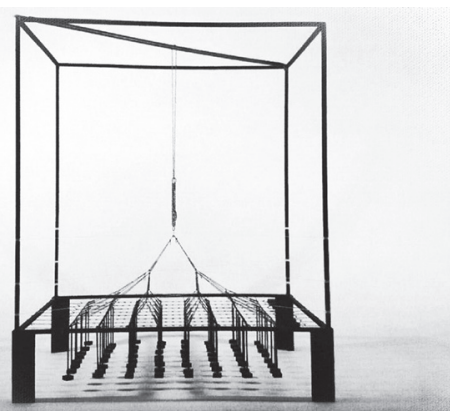

[5]

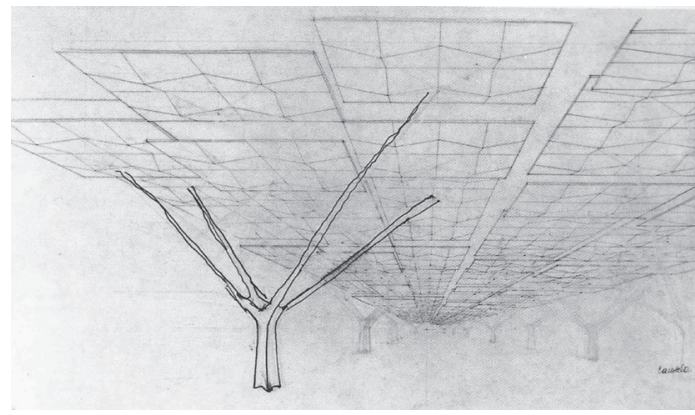

[6]

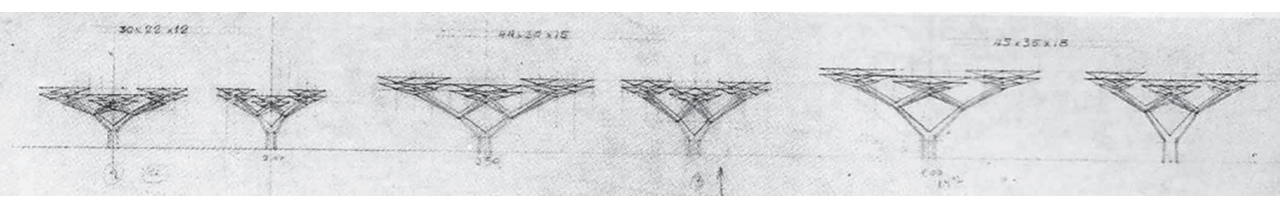

[7]

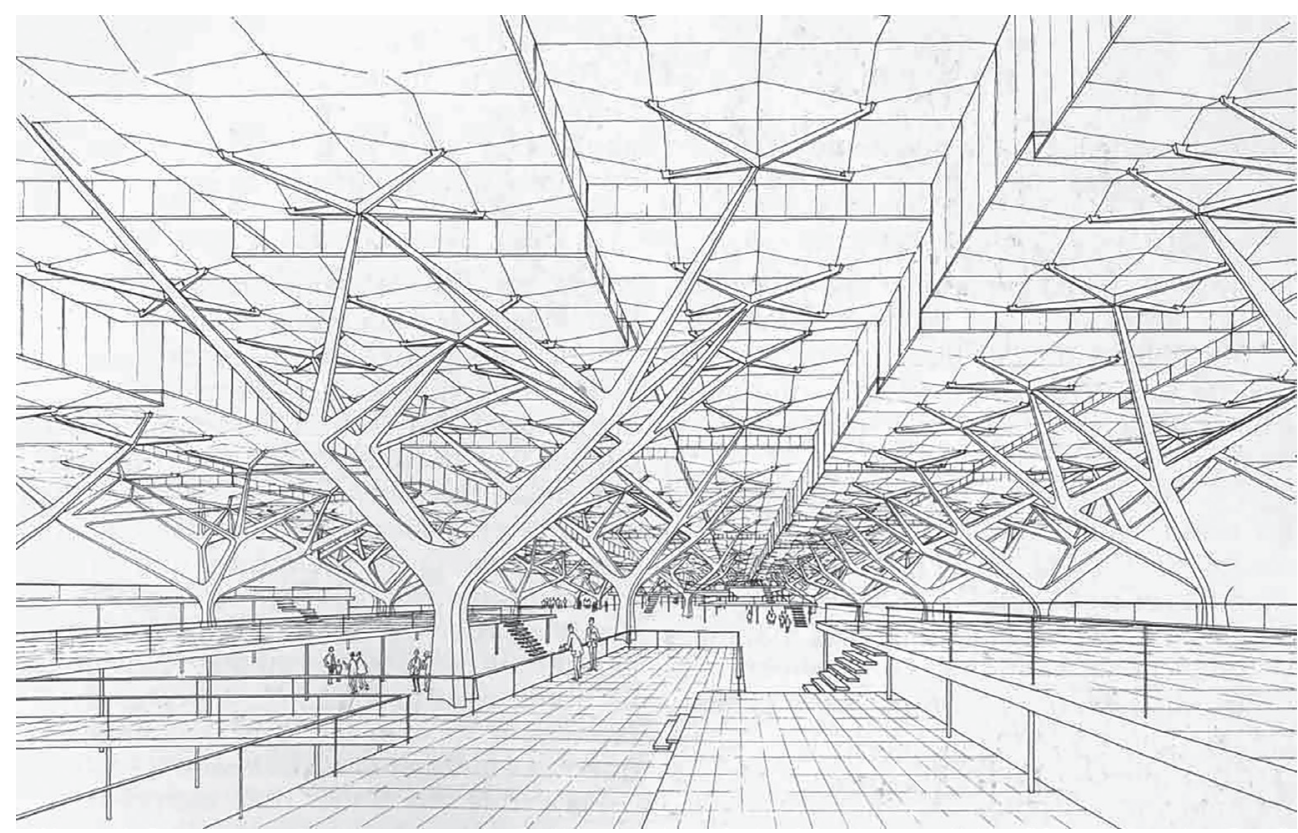

[4] Estructura arbórea de Frei Otto, 1960, catálogo de estudios experimentales presentado por el MOMA en 1972. NERDINGER, Winfried. Frei Otto. Complete Works. Swizerland: Birkhaüser, 2005, p 67.

[5] Perspectiva cónica interior para el concurso del Crystal Palace, Londres 1964. SEGU BUENAVENTURA, Miguel. Félix Candela arquitecto: exposición, depósito Elevado del Canal de Isabel II. Madrid: MOPTMA, Instituto Juan de Herrera, 1994

[6] Alzados de los módulos estructurales para el concurso del Crystal Palace, Londres 1964 Planos originales de Félix Candela. SEGUÍ BUENAVENTURA, Miguel. Félix Candela arquitecto: exposición, depósito Elevado del Canal de Isabel II. Madrid: MOPTMA, Instituto Juan de Herrera, 1994

[7] Perspectiva cónica interior de la sala de exposiciones para el concurso del Palacio de los Deportes de México, 1968. CASSINELLO Pepa. Félix Candela centenario 1910-2010. Madrid: Fundación Juanelo Turriano, 2010. 
En palabras de Félix Candela (1972), "de entre los muchos proyectos presentados, había uno realmente extraordinario, al que, naturalmente, concedimos el premio sin mayor discusión. Su autor era Emilio Pérez Piñero, entonces estudiante de la escuela de Madrid" ${ }^{2}$. Emilio Pérez Piñero presentó un teatro transportable capaz de plegarse mediante una cúpula según un sistema de su propia invención.

Este fue el primer contacto entre Félix Candela y Emilio Pérez Piñero quien desempeñará un papel fundamental en las cúpulas que diseñará Félix Candela en los años posteriores. La estrecha colaboración entre ambos arquitectos culminará en el año 1972 con el proyecto para la cúpula del velódromo de Anoeta. [8]

\section{Antecedentes del proyecto}

En 1961 Candela, quien ya conocía a Fuller desde 1957, quedó fascinado con el proyecto que presentó Emilio Pérez Piñero. Era consciente de las posibilidades que ofrecían las estructuras reticulares metálicas, pero por aquel entonces su empresa Cubiertas Ala funcionaba a pleno rendimiento, sus estructuras laminares se estaban publicando en todo el mundo, y seguía explorando las posibilidades geométricas del paraboloide hiperbólico.

Además las estructuras metálicas tenían un menor coste económico que las estructuras laminares de hormigón en un país como México.

Esta situación dio un giro inesperado con la alteración de los salarios mínimos en 1964, lo que trastocó sus sistemas de construcción encareciendo la realización de sus artesanales encofrados. Su reacción no se hizo esperar y ese mismo año presentó una estructura reticular metálica como solución para el concurso del Crystal Palace de Londres.

Para llevar a cabo su nuevo proyecto Candela se basó en el diseño de estructuras que ya conocía. Fusionando en un único diseño dos tipos diferentes de estructuras que ya habían sido realizadas, obtuvo como resultado un novedoso sistema formado por una estructura reticular metálica que soportaba un cerramiento de paraboloides hiperbólicos:

- La cubierta sigue la geometría de la estructura de paraguas invertido que llevaba construyendo desde hacía años. Candela ya conocía la eficacia de este tipo de estructuras. Fue él la primera persona que construyó una cubierta con esta geometría, un diseño que con sus propias palabras definió como su aportación más importante a la arquitectura, debido a su posibilidad de repetición, bajo coste y alto rendimiento.

- Los soportes -en forma de estructura arbórea- se inspiran en un diseño que Frei Otto había realizado para una exhibición en la Universidad de Yale en 1960. Hasta el momento Félix Candela no había experimentado con estructuras metálicas, y mucho menos con complejos sistemas espaciales de crecimiento ramificado. Sin embargo, Frei Otto -amigo de Félix Candela- era una de las figuras más representativas del panorama internacional en el campo del diseño e innovación de estructuras. La credibilidad de Otto resultó suficiente para que Candela confiara en este sistema.

El nuevo diseño realizado por Félix Candela no ganó el concurso para el Crystal Palace y el proyecto no se llegó a construir. Sin embargo, Candela volverá a proponer esta solución para el edificio anexo al estadio olímpico de México de 1968.

Tres años antes, en 1965, Candela recibió una llamada de Mr. Cutting ofreciéndole realizar un proyecto de instalaciones deportivas para la universidad de Brown. La propuesta incluía asociar su nombre con el de Mr. Cutting y Praeger-Kavanagh-Waterbury para formar la nueva asociación Cutting-Praeger-Candela.

Según el arquitecto (1994): "Un día me llamó por la mañana a México, a las siete de la mañana o cosa así. Me dice, soy Mr. Cutting un arquitecto, tengo un cliente, Mr. Nicolas Brown (...) que quiere traer o a Nervi o a usted para hacer un proyecto. Me dice, y como está usted más cerca le llamo a usted, por si quiere asociarse conmigo y con una empresa de Nueva York. Y así salió la asociación" 3 .

Esta propuesta de asociación no podía llegar en mejor momento con Félix Candela abierto a nuevas alternativas de experimentación y el incierto futuro de las estructuras laminares.

Tanto Nervi como Candela tenían un perfil claramente estructuralista, y la asociación de ingenieros Praeger-Kavanagh-Waterbury acababa de construir Astrodome, la mayor cúpula del mundo que salvaba 212 metros de luz mediante una estructura reticular metálica que cubría
2 CANDELA, Félix. Julio 1972. 'Emilio Pérez Piñero' Revista Nacional de Arquitectura, p. 9. Madrid.

${ }^{3}$ DE GARAY, Graciela. Grabación de sonido. Entrevista al arquitecto Félix Candela realizada en su domicilio en Riley, North Carolina. 9 agosto 1994 
4 "Ese proyecto duró varios años, y no se llegó a hacer nada. Porque a la vez que se terminaba el proyecto, hacíamos el presupuesto y resultaba que era demasiado dinero. Entonces decia, -hágamelo un poco más pequeño-; se lo haciamos más pequeño, $y$ como había subido el coste de la vida tambié se le salia el presupuesto. Total, no hicimos nada, pero sí estuvimos trabajando varios años con la empresa ésta de Nueva York. Y ese es uno de los motivos por los que yo sali de México, porque tenía ya trabajo en Nueva York y quería ver si podía liberarme de la cosa de contratista, de los problemas económicos de la empresa... Vivir de una manera más libre". DE GARAY, Graciela. Grabación de sonido.

Entrevista al arquitecto Félix Candela realizada en su domicilio en Riley, North Carolina. 9 agosto 1994 .

5 El proyecto para la cubierta del velódromo de Anoeta supuso el último proyecto que realizó Emilio Pérez Piñero y la última cúpula en la que participaría Félix Candela. Los detalles de la colaboración para el proyecto han sido detallados por $\mathrm{M}^{\mathrm{a}}$ Carmen Pérez Almagro (2013) en su tesis doctoral, Estudio y normalización de la colección museográfica y del archivo de la Fundación Emilio Pérez Piñero y por Migue Segui (1994) en el catálogo de la exposición 'Arquitecturas Ausentes del siglo XX'

${ }^{6}$ A raíz de este concurso, la fama de Emilio Pérez Piñero y sus novedosos diseños comenzaron a expandirse. Tal y como explicaba Salvador Dali, quería que Fuller realizara una de sus cúpulas para la construcción del Teatro Museo Dalí. Sin embargo, en conversación con B. Fuller, este le comentó: "tiene Vd. a Piñero que hace cosas que yo no sé cómo las hace", convenciendo a Dali de que fuera el joven arquitecto de Calasparra el que realizara el proyecto en lugar del propio Fuller. Transcripción de la entrevista realizada por Carlos de Miguel a Salvador Dalí.

${ }^{7}$ En una carta dirigida a Félix Candela, Emilio Pérez Piñero denuncia cómo para la realización de la órbita del Apolo 11 plagiaron uno de sus diseños. Por miedo a que volvieran a copiar sus patentes, Piñero se negó a

mandar maquetas de sus modelos a Estados Unidos, lo que dificultó la venta de las patentes. Fuente: depósito de la fundación Emilio Pérez Piñero, $n^{\circ}$ de referencia: ES_FEPP_ S1 0300056. la totalidad de un estadio de beisbol. Quedaba claro cuáles serían las intenciones de la nueva asociación a la que Félix Candela no dudo en unirse: Cutting-Praeger-Candela.

Para Candela suponía la oportunidad no solo de volver a construir en Estados Unidos, sino también de colaborar con especialistas en estructuras metálicas. Suponía además la oportunidad de entrar en un nuevo mundo: el de la geometría esférica de cúpulas para salvar luces inimaginables.

Los proyectos para las instalaciones de la universidad de Brown comenzaron en 1965 y se prolongarán hasta aproximadamente 1972 cuando se disuelve la asociación. Se realizaron múltiples propuestas ${ }^{4}$.

A pesar de que no se construyera ninguna, estas propuestas suponen el antecedente inmediato de la cúpula del Palacio de los Deportes de México basada en una estructura metálica de arcos esféricos con un cerramiento de paraboloides hiperbólicos. [9]

Esto supone a su vez el comienzo de una nueva línea de actuación marcada por estructuras cupulares para salvar grandes luces sobre las que se genera un cerramiento formado por la combinación de paraboloides hiperbólicos. Un diseño de cúpulas generado a partir de su geometría y no mediante cálculos analíticos.

En los años posteriores a la construcción del Palacio de los Deportes de México, Candela presentará nuevas alternativas de cúpulas para las instalaciones deportivas de la Universidad Brown. En 1969 competirá frente a Frei Otto y Nervi en el concurso para la ciudad deportiva de Kuwait donde volverá a proponer una cúpula con un cerramiento parabólico hiperbólico. Y finalmente en 1972 realiza para el velódromo de Anoeta el proyecto de su última cúpula ${ }^{\mathbf{5}}$, continuando un proyecto que comenzó Emilio Pérez Piñero.

No podemos pasar al siguiente punto sin hacer mención de la importancia de la relación que se produjo entre Candela y Emilio Pérez Piñero durante estos años. El primer contacto entre ambos arquitectos se fecha en 1961, con motivo del mencionado concurso que ganó Pérez Piñero y que tan gratamente sorprendió a Candela y Fuller ${ }^{6}$.

No fue hasta el 18 de junio de 1968 cuando ambos se volverían a encontrar por mediación de Carlos de Miguel. Piñero tenía intención de viajar a México y Estados Unidos con el fin de colocar sus patentes, y por aquel entonces Candela -que ya contaba con gran prestigio internacional- podría emplear sus contactos. Entre los interesados por las estructuras desplegables de Piñero se encontraban el Departamento de la Armada de Washington y la NASA ${ }^{7}$. Finalmente no consiguieron entrar en el mercado estadounidense, pero sí materializaron su asociación abriendo una oficina en Madrid. Desde 1968 a 1972 realizaron varios proyectos en común, sin que ninguno llegara a construirse.
[9]

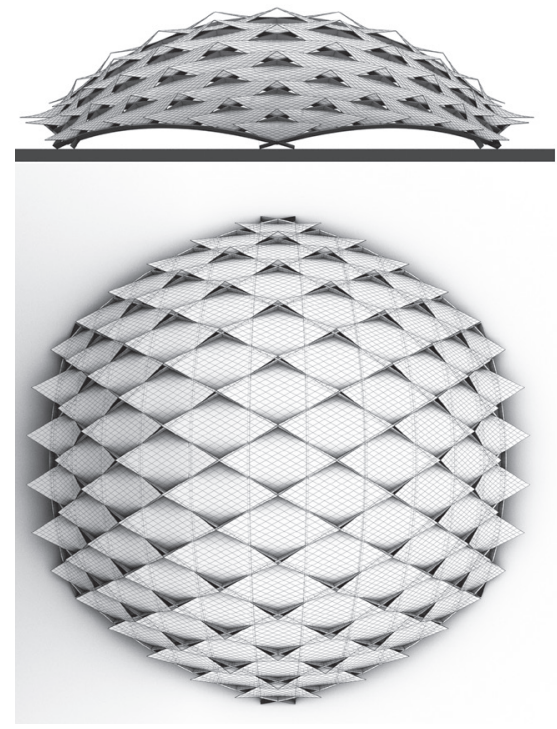

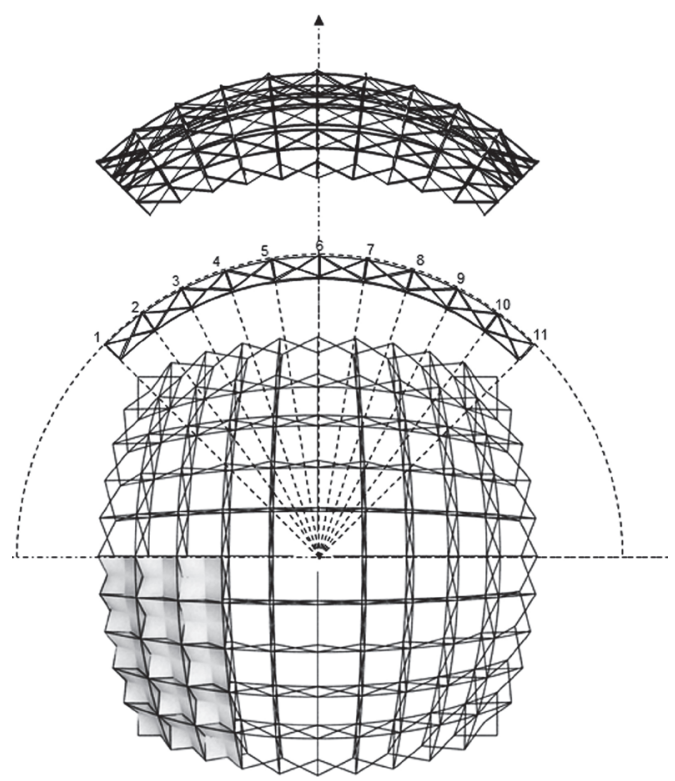

[8] Reconstitución de la cubierta para el velódromo de Anoeta de Emilio Pérez Piñero y Félix Candela, 1972. Imagen del autor, 2015.

[9] Esquema de la cubierta del Palacio de los Deportes de México, 1968. Aproximación de la geometría. Dibujo inédito realizado y cedido por Carmen García Reig, 2009. 
En paralelo a la asociación, cada uno llevaba proyectos de manera independiente. Coincidiendo con los años de la asociación, Candela se centró en el desarrollo de grandes cúpulas con cerramiento parabólico hiperbólico. La correspondencia mantenida entre ambos deja constancia del asesoramiento que Piñero -especializado en estructuras reticulares metálicas- dio a Candela para el diseño de sus cúpulas.

En 1972, mientras Piñero se encontraba sumido en el concurso para la cubierta del velódromo de Anoeta, fallece en un trágico accidente. En ese mismo año la asociación entre Candela y Praeger-Kavanagh-Waterbury se disuelve y Félix Candela abandona definitivamente el mundo de las estructuras cupulares.

\section{Documentación original}

Se presentan a continuación los planos originales realizados por Félix Candela para el concurso del Palacio de los Deportes -bajo el lema "corpore sano"- en los que aparece junto a la gran cúpula construida el edificio anexo para exposiciones y ferias.

La documentación original de Candela está recogida principalmente en el Archivo de la Avery Architectural and Fine Arts Library. A finales de los años 80 Félix Candela donó a la universidad de Columbia la mayor parte de sus archivos, incluyendo planos y correspondencia. Algunos de los planos referentes al Palacio de los Deportes de México fueron publicados por Miguel Seguí para la exposición realizada en honor a Félix Candela, en el depósito Elevado del Canal de Isabel II.

Existen a su vez otras dos fuentes documentales de menor importancia: los archivos de la universidad de Princeton -cedidos por Dorothy Candela-, y los documentos que se encuentran en la universidad nacional autónoma de México -donados por las hijas de Félix Candela-.

La fundación Emilio Pérez Piñero posee los archivos referentes a la colaboración entre ambos arquitectos, a excepción de la maqueta para el velódromo de Anoeta, que se trasladó a Columbia.

Finalmente, existen dos fondos documentales -inéditos- en España referentes a los últimos años de la obra de Félix Candela, posteriores a la donación realizada a la Universidad de Columbia. [10] [11] [12] [13]

\section{Geometría y modulación}

Desde 1951 la obra de Félix Candela está caracterizada por la utilización de la geometría del paraboloide hiperbólico y sus diferentes combinaciones para generar las cubiertas de sus proyectos.

La estructura de paraguas invertido que Candela construyó por primera vez en el año 1952 estaba formada por cuatro fragmentos de paraboloides hiperbólicos simétricos que se apoyaban sobre un único soporte vertical que transmitía sus cargas al terreno. Durante más de una década se convirtió en la estructura más económica que se realizaría en México.

[10]

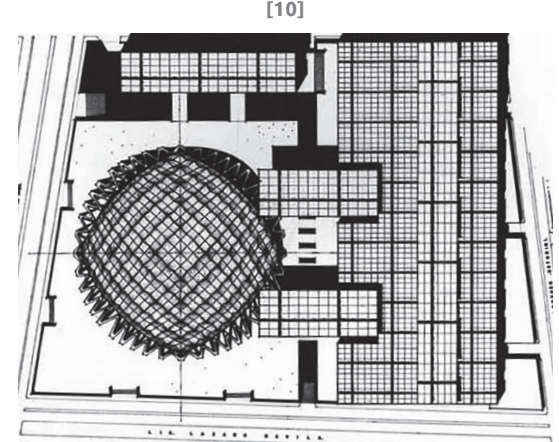

[12]

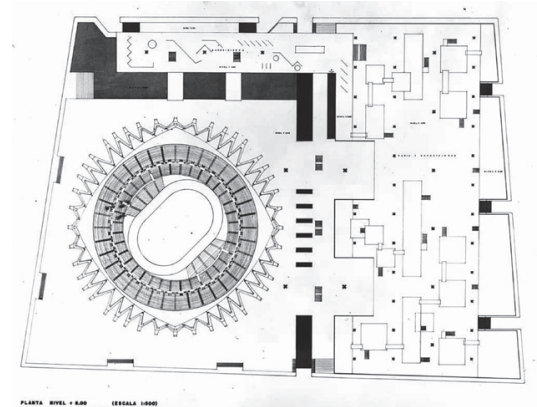

$=-\cdots$
[10] [11] [12] [13] Plantas de la cúpula y la sala de exposiciones. Planos originales de Félix Candela presentados al concurso del Palacio de los Deportes para las Olimpiadas de México. Archivo de la Avery Architectural and Fine Arts Library. 1968.

[14] Perspectiva axonométrica. Reconstitución del módulo de la sala de exposiciones para el Palacio de los Deportes de México, 1968. Imagen del autor, 2015.

[15] [16] Perspectivas cónicas. Reconstitución del módulo de la sala de exposiciones para el Palacio de los Deportes de México, 1968. Imágenes del autor, 2015.

[17] Planta fugada. Reconstitución del módulo de la sala de exposiciones para el Palacio de los Deportes de México, 1968. Imagen del autor, 2015.

[18] Planta invertida. Reconstitución del módulo de la sala de exposiciones para el Palacio de los Deportes de México, 1968. Imagen del autor, 2015.

[19] Perspectiva cónica del conjunto. Reconstitución 3D. Imagen del autor, 2015 


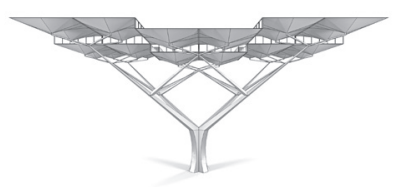

[15]

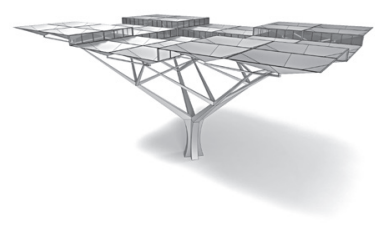

[16]

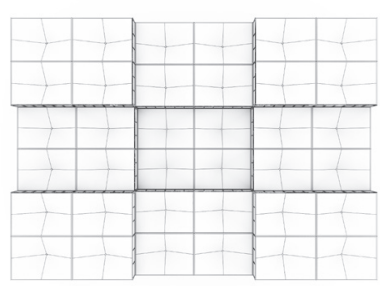

[17]

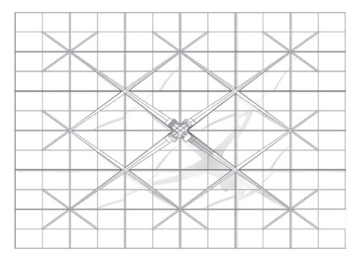

[18]
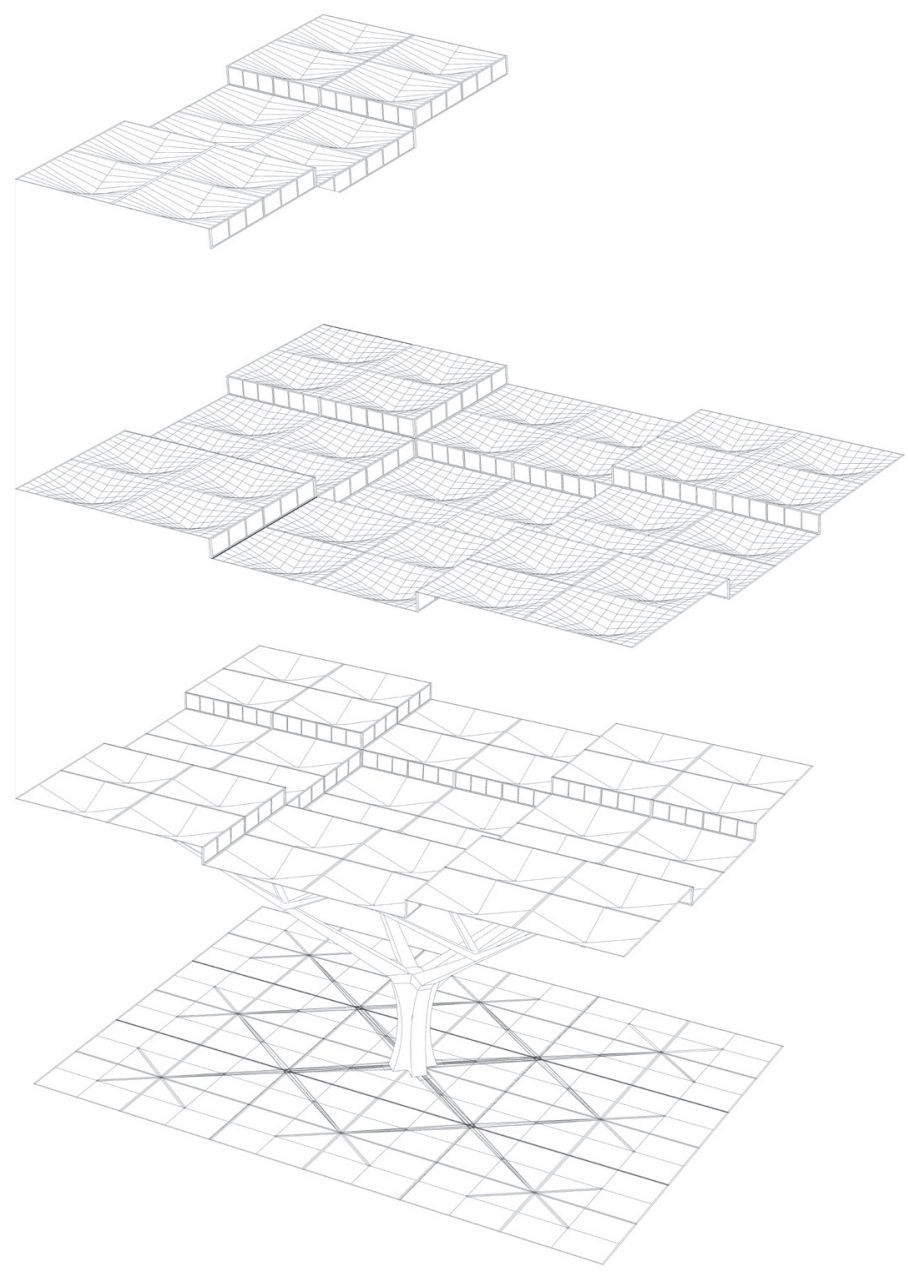

[14]

Candela vuelve a repetir su estructura más famosa en la sala de exposiciones del Estadio Olímpico de México proyectando la cubierta según una composición de estos paraguas. [14]

Las dimensiones de cada paraguas son de 7 × 5 metros en planta, luces habituales para este tipo de estructura y que en proyectos previos Candela ya había superado ampliamente.

La diferencia principal radica en los soportes, ya que la sencillez del pilar del paraguas original es sustituida aquí por una compleja estructura arbórea que se ramifica progresivamente para sostener 36 paraguas invertidos permitiéndole cubrir un área de $42 \times 30$ metros. [15] [16]

Los fragmentos de hypar generan un paraguas invertido que se agrupa con otros tres a la misma cota. El módulo principal es una matriz rectangular de 9 de estos grupos a alturas distintas para permitir el paso de la luz natural al interior. [17] [18]

Para generar la cubierta completa los módulos se yuxtaponen uno a continuación de otro. La proyección en planta de cada módulo genera un rectángulo que, repetido, compacta la planta.

Se consigue un espacio único y sin particiones. Los componentes estructurales aparecen como elementos verticales que no segmentan el espacio y que marcan un ritmo repitiéndose cada 42 metros longitudinalmente y cada 30 transversalmente. [19]

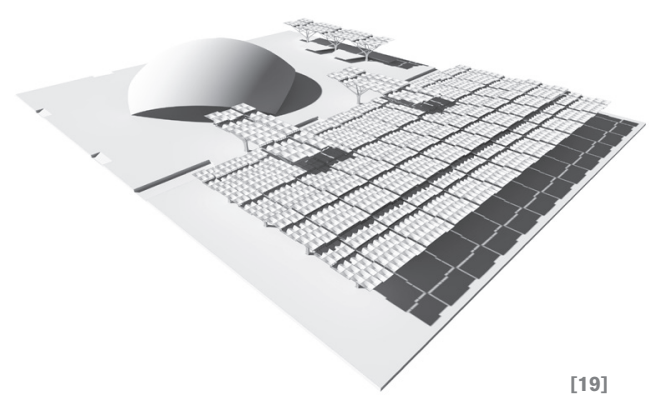

[19] 
El proyecto se percibe desde el interior como un bosque de ramas metálicas. Resulta complicado para el espectador diferenciar dónde acaba y empieza cada módulo debido al juego en la variación de altura de los paraboloides de cubierta. A pesar de seguir un ritmo modulado, la cubierta se percibe como un único elemento. Los límites entre los diferentes módulos no existen y son los soportes los elementos que permiten tener una referencia del módulo original.

\section{Soportes ramificados}

Como ya hemos mencionado, Félix Candela toma como referencia los diseños de Frei Otto para generar el diseño estructural de los soportes. A diferencia de los diseños de Frei Otto, Candela opta por generar un crecimiento simétrico de las ramas, de manera que de cada nudo surgen cuatro nuevas ramas simétricas entre sí. [20]

Se trata de una solución coherente que simplifica el diseño original de Frei Otto, asegurándose que el reparto de las cargas en los nudos sea siempre proporcional para cada nueva ramificación.

A pesar de que la documentación original de Félix Candela no permite reconstituir en detalle el sistema constructivo ni la evacuación de aguas para asegurar la estanqueidad de la cubierta, un estudio de la obra de Candela indicaría que los soportes serían huecos para evacuar el agua de la cubierta. Aprovechando la inclinación de los paraboloides hiperbólicos, el agua se vertería hacia su interior. [21]

\section{Iluminación natural}

El tratamiento de la luz natural ha sido siempre un tema esencial en la arquitectura de Félix Candela. Los recortes en la geometría de sus cubiertas parabólico-hiperbólicas quedan condicionados por la manera de iluminar los interiores, lo que confiere una particularidad a cada proyecto.

En el proyecto que estamos analizando la necesidad de iluminar la gran superficie que cubre cada módulo condiciona la disposición de los grupos de hypar. Candela decide situar estos grupos a diferente cota para conseguir huecos horizontales por los que la luz penetre al interior. Evita de esta manera inclinar los diferentes paraguas o realizar perforaciones sobre la superficie de cubierta; opciones que había utilizado en proyectos anteriores. [22] [23]

Como es frecuente en los proyectos de Candela, el espacio interior es único, sin particiones y con una gran altura desde el suelo a la cubierta, lo que permite que la luz se extienda sin barreras iluminando todo el interior. La incidencia de la luz directa que se rompe sobre las barras de las estructuras de los soportes y las sombras que provoca potencian todavía más la percepción de una cubierta iluminada como un bosque.

\section{El límite}

El tratamiento del límite es diferente en cada proyecto de Félix Candela. Los voladizos, la esbeltez de sus cubiertas así como las grandes luces que salva han concedido gran importancia a este aspecto en la obra de Candela. [24]

En la sala de exposiciones del Palacio de los Deportes de México la estructura arbórea determina que desde el soporte central hasta los bordes del módulo haya una distancia de 21 y 15 metros.

No existe un límite físico ni visual, el perímetro es completamente abierto. El gran voladizo de 21 metros sirve como espacio de transición entre el exterior y el interior de forma que el caminante podría penetrar hacia el interior del edificio sin ningún impedimento ni obstáculo.

Desde el exterior se puede apreciar el proyecto completo al no existir tampoco ningún tipo de partición interior. Muy notable de señalar también en la sala de exposiciones es que, dentro de este gran espacio unificado, los forjados se proyectan a diferentes alturas, algo inédito en la obra de Candela. [25]

\section{Conclusiones}

El Palacio de los Deportes de México de 1968 fue el último gran proyecto que Félix Candela construiría. Tanto la cúpula como la sala de exposiciones anexa analizada y dibujada en este artículo muestran un cambio en la trayectoria del arquitecto dedicada casi exclusivamente al desarrollo de estructuras laminares empleando la geometría del paraboloide hiperbólico. Por diversas circunstancias, Félix Candela prácticamente no volvería a construir más proyectos, quedando el Palacio de los Deportes como una obra singular en su larga trayectoria.

[20] Crecimiento según el número de iteraciones de una estructura ramificada. Imagen del autor, 2015.

[21] Sistema de evacuación de agua a través del soporte en una estructura estándar de paraguas invertidos. FABER, Colin. Candela, the Shell builder. London: Architectural Press, 1963

[22] [23] Perspectiva cónica interior de la sala de exposiciones. Imagen del autor, 2015

[24] Perspectiva cónica exterior de la sala de exposiciones. Reconstitución tridimensional. Imagen del autor, 2015.

[25] Alzados y secciones de la cúpula y la sala de exposiciones. Planos originales de Félix Candela presentados al concurso del Palacio de los Deports para las Olinpiada Palacio de los Deportes para las Olimpiadas de México. Archivo de la Avery Architectural and Fine Arts Library, 1968 
A través de una investigación realizada acerca de la arquitectura no construida de Félix Candela, hemos podido llegar a la conclusión de que ambos proyectos no son un caso aislado, sino el comienzo de una nueva línea de actuación que se desarrollaría en los años posteriores. Queda abierta una vía de investigación para la recuperación y reconstitución gráfica de proyectos inéditos en la obra de Candela así como para el análisis de geometría y estructuras reticulares metálicas.

[20]

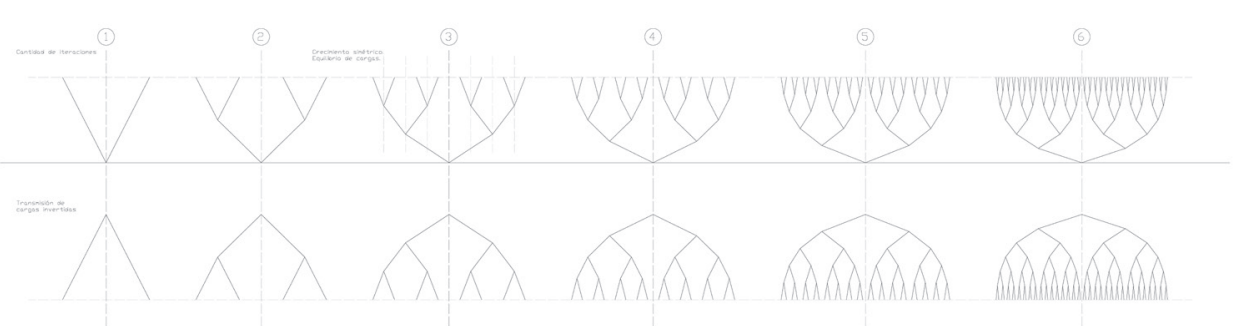

[22]

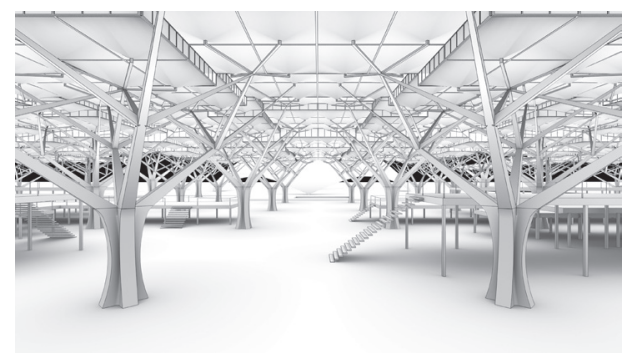

[23]

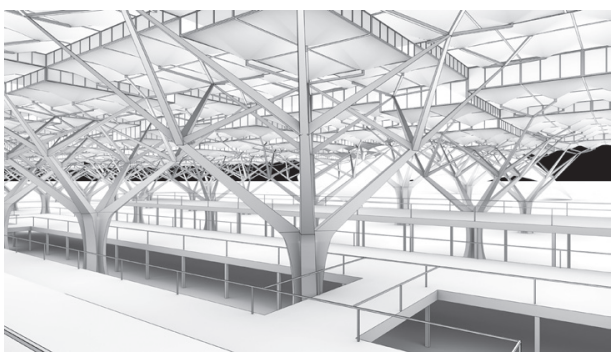

$[25$
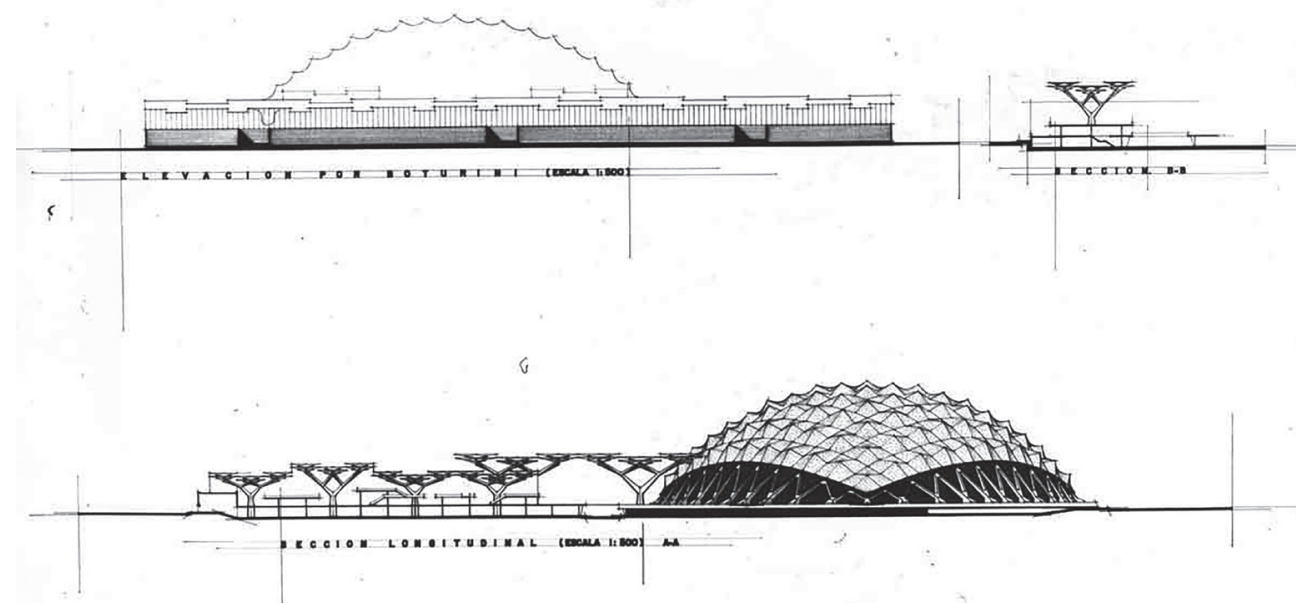
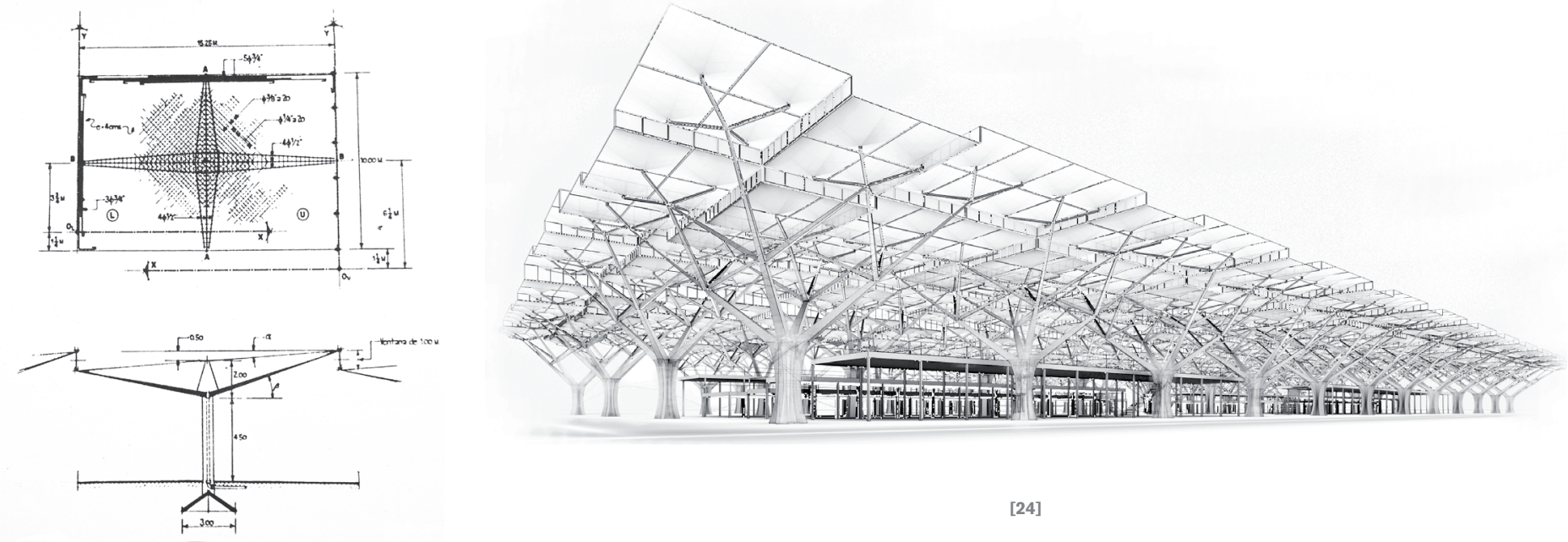


\section{Resumen 05}

El trabajo que se expone a continuación es parte del resultado de un trabajo de investigación realizado en la Universidad Politécnica de Madrid. A través de este artículo, se analiza el proyecto de la sala de exposiciones para el concurso de Palacio de los Deportes de México realizado por Félix Candela en asociación con Enrique Castañeda y Antonio Peiri en el año 1968.

En el artículo se incluye la documentación original conservada en los depósitos de la Avery Architectural and Fine Arts Library y Princeton University Library, así como los planos e infografías inéditos, realizados por los autores de este artículo para la reconstitución del proyecto.

La nueva documentación generada adquiere especial relevancia al tratarse de un proyecto que no llegó a construirse y del que existe escasa información. A partir de ella hemos podido analizar la compleja geometría de la cubierta generada a partir de la combinación de paraboloides hiperbólicos que se apoyan sobre una estructura arbórea metálica.

A su vez, en el artículo se estudian las influencias que Candela tuvo por parte de arquitectos como Frei Otto o Emilio Pérez Piñero, que contribuyeron al cambio de rumbo que tomó su arquitectura abandonando sus icónicas estructuras laminares de hormigón para pasar a realizar estructuras metálicas.

\section{Abstract 05}

The work presented below is part of the results of a research project at 'Universidad Politécnica de Madrid'. In this paper we analyze the project of the exhibition hall next to the Sport Palace in México. It was designed by Felix Candela in association with Enrique Castañeda and Antonio Peiri in the year 1968.

The original plans made by Félix Candela are included in the research paper. They are stored in the Avery Architectural and Fine Arts Library and in the Princeton University Library. We have also added computer generated images and drawings, which allowed us to analyze the geometry of the project.

The new documents drawn by the authors of the paper are especially important since the project wasn't built. They allowed us to analyze the complex geometry of the project, which was done using hyperbolic paraboloids on branched pillars.

In the paper we study the influences that Felix Candela had from the architects Fre Otto and Emilio Pérez Piñero. Both of them contributed towards the new direction of Felix Candela's architecture.

\section{Bibliografía_Bibliography}

BASTERRA OTERO, Luis Alfonso. Las estructuras arquitectónicas de Félix Candela: Una revisión actual. Tesis doctoral, ETSAV, departamento de construcción. Valladolid, 1998

CANDELA, Félix. 'Arquitectura y estructuralismo. Arquitectura', n59, Noviembre 1963

CANDELA, Félix. En defensa del Formalismo y otros escritos. Bilbao: Xarait Ediciones, 1985.

CANDELA, Félix. A-246. Una estructura reticulada: el Palacio de los Deportes de México. I Encuentro Internacional de Estructuras Ligeras de Grandes Lucas. Fundación E. Pérez Piñero. Murcia 1992.

CANDELA, Félix. 07/72 Madrid. A-195. Emilio Perez Piñero. Leído en el acto en memoria de Emilio Perez Piñero que se celebró en el Instituto de la Vivienda Madrid, Arquitectura 1972. Obituario. Incluido en Xarait Ediciones. Bilbabo, 1985.

CASSINELLO, Pepa. Félix Candela centenario 1910-2010. Madrid: Fundación Juanelo Turriano, 2010.

CASSINELLO, Pepa. Félix Candela, la conquista de la esbeltez. Ayuntamiento de Madrid, 2010.

CUETO, José Ignacio del. Félix Candela 1910-2010. IVAM, SECC, 2010

FABER, Colin. Candela, the Shell builder. London: Architectural Press, 1963

SEGUí BUENAVENTURA, Miguel. Félix Candela arquitecto: exposición, depósito Elevado del Canal de Isabel II. MOPTMA, Instituto Juan de Herrera. Madrid, 1994.

Seguí BuenaVentuRA, Miguel. Félix Candela y Emilio Pérez Piñero: un diálogo imaginal. Ministerio de vivienda. 2005.

NERDINGER, Winfried. Frei Otto. Complete Works. Swizerland: Birkhaüser, 2005.

PÉREZ ALMAGRO, Ma Carmen. Estudio y normalización de la colección museográfica y del archivo de la Fundación Emilio Pérez Piñero. Tesis doctoral, Universidad de Murcia, departamento de prehistoria, Arqueología, historia antigua, Historia medieval y ciencias y Técnicas historiográficas. 2013.

PÉREZ PIÑEIRO, Emilio. 'Estructuras reticulares tridimensionales'. Arquitectura nº 112, abril 1962. 